\section{Effektive Therapiestrategien bei metastasiertem Nierenzellkarzinom}

\begin{abstract}
- Patienten mit einem metastasierten Nierenzellkarzinom (mRCC) profitieren von einer Kombinationstherapie mit dem Angiogenesehemmer Bevacizumab (Avastin ${ }^{\circledR}$ ) und IFN- $\alpha$. Der therapeutische Nutzen zeigt sich sowohl bei Patienten mit geringem als auch mit mittlerem Motzer-Risiko, bei älteren Patienten und bei mRCC-Patienten mit gemischter Histologie, berichtete Dr. Dirk Arnold, Halle. In der AVOREN-Studie führte die Kombinationstherapie im Vergleich zu der bisherigen Standardtherapie mit IFN zu einer Verdoppelung des progressionsfreien Überlebens (10,2 vs. 5,4 Monate, $\mathrm{p}<0,0001)$ und zu einer Erhöhung der Ansprechrate (31 vs. 13\%, p<0,0001) [Escudier B et al. Lancet 2007; 370: 2103-11]. Patienten, die eine First-Line-Therapie mit Bevacizumab/IFN- $\alpha$ erhalten hatten und nach einer Progression mit einem Tyrosinkinasehemmer in der Second-Line weiterbehandelt wurden, erreichten ein medianes Gesamtüberleben von mehr als 3 Jahren $(38,6$ Monate, $\mathrm{HR}=0,8)$.

Bei sehr hohem Progressionsrisiko profitieren mRCC-Patienten von einer FirstLine-Therapie mit dem mTOR-Inhibitor Temsirolimus (Torisel ${ }^{\circledR}$ ), so PD Dr. Thomas Steiner, Jena.Wenn man die Hudes-Kriterien
\end{abstract}

für die Risikostratifizierung zugrunde lege, gehörten etwa 39\% der mRCC-Patienten dem Hochrisikokollektiv an. Im Vergleich zu IFN verlängert Temsirolimus das Gesamtüberleben um 49\% (7,3 vs. 10,9 Monate, $\mathrm{p}=0,008$ ) [Hudes G et al. N Engl J Med 2007; 356: 2271-81]. Die gute Verträglichkeit des mTOR-Inhibitors schlägt sich auch in einer höheren Lebensqualität wieder. Die Zeit ohne Symptome und Toxizität (TWIST) war in der ARCC-Studie um 38\% langer als unter einer IFN- $\alpha$-Therapie (6,5 vs. 4,7 Monate). Das günstigere Toxizitätsprofil komme insbesondere Patienten mit fortgeschrittener Erkrankung und eingeschränkter körperlicher Belastbarkeit zu Gute, betonte Steiner. Subgruppenanalysen der ARCC-Studie ergaben, dass Patienten unabhängig von dem Nephrektomiestatus von Temsirolimus profitieren, also auch dann, wenn ihnen keine Operation mehr zugemutet werden kann [Logan T et al. J Clin Oncol 2008; 26 (Suppl): Abstract 5050].

aam

Satellitensymposium „Aktuelle Therapie des Nierenzellkarzinoms mit Avastin ${ }^{\circledR}+$ IFN und Torisel ${ }^{\circledR}$ "Im Rahmen des DGU-Kongresses, Dresden, 17. September 2009; Veranstalter: Roche und Wyeth Pharma

\title{
Überlebensvorteil beim Urothelkarzinom
}

_ Ohne Behandlung haben Patienten mit fortgeschrittenem oder metastasiertem Urothelkarzinom nach dem Versagen einer platinhaltigen Chemotherapie eine Lebenserwartung von weniger als sechs Monaten. Mit Vinflunin (Javlor ${ }^{\circledR}$ ) gibt es nun eine effektive Therapieoption, erklärte Dr. Joaquim Bellmunt, Barcelona. Ende Juni 2009 war die Marktzulassung der antineoplastischen Substanz als Monotherapie zur Behandlung des metastasierten Harnblasenkarzinoms (fortgeschrittenes oder metastasiertes Übergangszellkarzinom des Urothels nach Versagen nach Versagen einer Platin-haltigen Therapie) empfohlen worden. Sie beruhte auf den Ergebnissen zweier Phase-IIStudien sowie einer Phase-III-Studie. In der randomisierte Phase-III-Studie war Vinflunin (VFL) plus Best Supportive Care (BSC) mit
BSC alleine verglichen worden [Bellmunt J et al. J Clin Oncol 2009; 27(27):4454-61].Die 249 Patienten im VFL+BSC-Arm lebten im Mittel um 2,6 Monate länger als die im BSCArm (6,9 vs. 4,3 Monaten). VFL hatte einen statistisch signifikanten Behandlungseffekt auf das Gesamtüberleben, so Bellmunt. Die Nebenwirkungen Verstopfung, Neutropenie und Fatigue waren akzeptabel, vorhersehbar und beherrschbar. Kumulative Toxizitäten traten nicht auf. Am 21. September 2009 erhielt Pierre Fabre die Zulassung. ut

Symposium „Javlor: Erste zugelassene Option nach platinhaltiger Therapie beim fortgeschrittenen oder metastasierten Urothelkarzinom", im Rahmen des DGU-Kongresses, Dresden, 18. September 2009; Veranstalter: Pierre Fabre Oncology

\section{Chondroitinsulfat bei} chronischen Cystitiden

Mehr als $76 \%$ der Patienten profitieren von Gepan ${ }^{\circledR}$ instill, so das Ergebnis einer Studie [Nordling J , van Ophoven A Arzneimittelforschung 2008;58(7):328-35]. Sie hatten bei 286 Patienten eine Glykosaminoglykan (GAG)-Ersatztherapie mit Chondroitinsulfat durchgeführt. Dass die GAG-Ersatztherapie auch bei überaktiver Blase zu einer Besserung der Symptomatik führt konnte Annett Bauruder-Burmester in zwei Studien belegen [Zentralbl Gynäkol 2006; 128:336 und Geburtsh Frauenheilk 2008; 68: 1077]. Seit 15. November bietet Pohl-Boskamp das Produkt auch als Fertigspritze an.

Nach Informationen von Pohl Boskamp.

\section{Astellas unterstützt den Verein Fistula e.V.}

Seit drei Jahren unterstützt die Astellas Pharma GmbH ein einzigartiges Programm in Äthiopien: Das Fistula-Projekt. Vesicovaginale Fisteln oder auch Geburtsfisteln sind in Entwicklungsländern ohne ausgeprägte medizinische Versorgung ein schwerwiegendes gesundheitliches aber auch soziales Problem. Auf dem DGU-Kongress wurde nun um Spenden ,gedartet" und gerudert. Für jedes zielgenaues Treffen der Dart-Scheibe im 20-iger-Feld oder für mehr als 30 Sekunden Rudern auf dem Ergometer spendete Astellas Pharma einen Betrag von 5 Euro. Die DDG-Besucher waren sehr aktiv. Insgesamt kamen 11.235 Euro an Spendengeld zusammen.

Nach Informationen von

Astellas Pharma.

\section{Flächendesinfektion} mit Dismozon ${ }^{\circledR}$

Clostridium difficile ist die häufigste Ursache nosokomialer Diarrhöen. Das Aktivsauerstoff-Produkt Dismozon ${ }^{\circledR}$ pur führt mit einer Konzentration von 1,5\% innerhalb von 2 Stunden zu einer sicheren Desinfektion bei Sporen von drei verschiedenen Costridium-difficile-Stämmen, darunter auch der besonders virulente Stamm Ribotyp 027.

Nach Informationen von Bode Chemie. 